\title{
Computer Algebra for the Formation of Structural Matrices of Piezoceramic Finite Elements
}

\author{
Algimantas Čepulkauskas, Regina Kulvietienè, and Genadijus Kulvietis \\ Vilnius Gediminas Technical University \\ Sauletekio 11, Vilnius 2040, Lithuania \\ \{algimantas_cepulkauskas, regina_kulvietiene, \\ genadijus_kulvietis\}@gama.vtu.It
}

\begin{abstract}
This paper deals with the description of a theoretical background of systematic computer algebra methods for the formation of structural matrices of piezoceramic finite elements. The efficiency of computer algebra application was compared here with the numerical integration methods of forming the structural matrices of the finite elements. To this end, the computer algebra system VIBRAN was used. Two popular finite elements for modelling piezoceramic actuators of the sector-type and the triangular one are discussed. All structural matrices of the elements were derived, using the computer algebra technique with the following automatic program code generation.
\end{abstract}

\section{Introduction}

Recent advances in the development, theory and applications of new smart materials, structures and devices, including the materials with extremely high piezoelectric or magnetostrictive properties (e.g., Terfenol-D or PMN - lead magnesium niobate) have extended the area of mechatronics, providing systems with very high levels of integration and multifunctionality [3. Some concepts, e.g., actuators with an infinite number of degrees of freedom, transmission of energy to actuators through some distance, active bearings, etc., could be treated as solutions that define a problem. In some cases, the introduction of piezomechanical systems creates a new synergistic effect and, as in all integrated systems, the problem of maximum interaction between subsystems is the key to optimal design. Although the introduction of new piezoactive materials, that have found application in the areas of actuators, transducer technology, energy transformers, control devices, etc., has been very intensive in recent years [1, 1, 12, 16], the main concepts, ideas and effects are relatively unknown to design engineers. The holistic complex interaction of dynamic effects, energy transformation and devices based on them are described, while the physics involved in the piezoelectric theory may be regarded as the coupling between Maxwell's equations of electromagnetism and the elastic stress equations of motion [9, 11, 13, 14].

The finite element method (FEM) is widely used for modelling complicated structures [17. In the FEM the continuum is digitized and thus the numerical 
approximation is chosen. By means of numerical integration the result of sufficient accuracy could often be obtained, and using more effective computer facilities, this technique is mostly chosen. However, the order of numerical integration implies thorough adjustment to the order of polynomial expressions to be integrated, and this fact is not generally known. The use of a more precise formula for numerical integration will not produce more accurate results, but will somewhat 'stiffen' the examined structure [5, 6]. On the other hand, even the use of the exact numerical integration scheme always yields some numerical error that can become important in particular finite element applications, requiring a high accuracy of analysis [10, 15].

Moreover, it is known that the formation of the structural matrices is one of the most computationally expensive procedures in FEM 11, and the use of numerical integration for these purposes is not the optimal solution. This fact becomes particularly keen in case of finite element applications in the structures of piezoactive materials. Besides the conventional stiffness matrix there appear three additional ones: matrices of mass, electro-elasticity, and capacity [1,4]. The numerical integration procedure is especially expensive for the formation of the latter matrices, because the power of integral expressions is higher. The computer algebra offers wide opportunities in the formation of finite element structural matrices. Regretfully we see that much routine algebraic work in the FEM, is usually done by hand or by means of numerical approximations, while it could be done by the computer algebra technique much faster, reliably and better. Another most important aspect is that the results, obtained in this way, often make considerable economies of computer resources and assure a proper accuracy of evaluations [5. Despite that, even the classical books on FEM [17] do not mention any possibility of symbolic manipulations in the formation of element structural matrices.

There is one advantage of the numerical integration mentioned: for a particular type of finite elements, the structural matrices are always to be expressed in the same way through interpolating functions and their derivatives, and therefore the universal computational subroutines can be applied to various finite elements 8, 17]. However, the same extent of generality is a characteristic feature of computer algebra methods, too. Moreover, the user of programs, generated by computer algebra, does not have to consider the powers of polynomial expressions to be integrated, which is necessary in order to select the proper numerical integration scheme [2]. Even the users of modern commercial codes (such as ALGOR, ANSYS, etc.) have determined the necessary number of integration points for some types of finite elements at the stage of initial data reading [5].

\section{Application of the Finite Element Method to Piezoceramic Structures}

The mechanical and electrical phenomena as well as their coupling complicate the application of FEM to piezoceramic structures, such as actuators or other devices mentioned above. Thus the state of each finite element nodal point can 
be represented by the values of the nodal displacement $\{\delta\}$ and potential $\{\varphi\}$ [1. When describing a finite element, the displacements and potentials at any point are expressed via nodal values as follows:

$$
\left\{\begin{array}{l}
\{U\}=[N]\{\delta\} \\
\{\Phi\}=[L]\{\varphi\}
\end{array}\right.
$$

where $[N]$ and $[L]$ are, in the general case, interpolating functions, and $\{\delta\},\{\varphi\}$ are nodal displacements and potentials, respectively.

The strain vector could be expressed in the form [17]

$$
\{\varepsilon\}=[B]\{\delta\}
$$

Similarly, the electric field could be expressed in the form [1]

$$
\{E\}=\left[B_{E}\right]\{\varphi\} .
$$

Thus, the equations of piezoelectric effect on the elementary volume are expressed as 16

$$
\left\{\begin{array}{l}
\{\sigma\}=\left[c^{E}\right]\{\varepsilon\}-[e]^{T}\{E\} \\
\{D\}=[e]\{\varepsilon\}+\left[\ni^{S}\right]\{E\}
\end{array}\right.
$$

where $\left[c^{E}\right],[e],\left[\ni^{s}\right]$ are the matrix of stiffness for a constant electric field, the matrix of the piezoelectric constant, and the matrix of the dielectric constant evaluated under constant strain, respectively; $\{\sigma\},\{\varepsilon\},\{\mathrm{D}\},\{\mathrm{E}\}$ are the vectors of stress, strain, electric induction, and the electric field, respectively.

The analysis of piezoelectric actuators must be made taking into consideration the electric occurrence in the system. Based on the FEM, each node of the element has an additional degree of freedom used for electric potentials in FEM modelling. The solution applied to the equations of motion, fit for the actuator, can be derived from the principle of the minimal potential energy by means of the variation functional 4. The basic dynamic FEM equation of motion for piezoelectric transducers that are entirely covered with electrodes can be expressed as follows [1]:

$$
\left\{\begin{array}{l}
{[M]\{\ddot{\delta}\}+[C]\{\dot{\delta}\}+[K]\{\delta\}-[T]\{\varphi\}=\{R(t)\},} \\
{[T]^{T}\{\delta\}+[S]\{\varphi\}=\{Q\},}
\end{array}\right.
$$

where $[\mathrm{M}],[\mathrm{K}],[\mathrm{T}],[\mathrm{S}],[\mathrm{C}]$ are the matrices of mass, stiffness, electro elasticity, capacity, and damping, respectively; $\{\delta\},\{\varphi\},\{R\},\{Q\}$ are the vectors of node displacements, potentials, external mechanical forces, and charges coupled on the electrodes, respectively.

Here the structural matrices of the piezoceramic finite element of volume $V$ are:

$$
[K]=\int_{V}[B]^{T}\left[c^{E}\right][B] \mathrm{d} V
$$




$$
\begin{aligned}
& {[T]=\int_{V}[B]^{T}[e]\left[B_{E}\right] \mathrm{d} V,} \\
& {[S]=\int_{V}\left[B_{E}\right]^{T}\left[^{s}\right]\left[B_{E}\right] \mathrm{d} V,} \\
& {[M]=\int_{V}[N]^{T} \gamma[N] \mathrm{d} V,} \\
& {[C]=\alpha[M]+\beta[K] .}
\end{aligned}
$$

The damping matrix $[\mathrm{C}]$ is derived using the mass and stiffness matrices by denoting the constants $\alpha$ and $\beta$. $\gamma$ is the mass density.

The procedure to obtain the structural matrices of finite elements consists of the following steps:

1. Definition of interpolating functions, which are inverse to the configuration matrix obtained from interpolating polynomials.

2. Obtaining the derivatives of interpolating functions.

3. Multiplication of all the necessary submatrices.

4. Integration of these products over the volume of a finite element.

\section{Implementation of Computer Algebra}

Usually piezoceramic actuators are of a simple form, e.g., plates, rings, cylinders, etc. 3, 7. Therefore the simple form finite elements are used. Two popular finite elements will be presented for modelling piezoceramic actuators of a sector-type and the triangular one. The interpolating functions for the sector-type finite element with four nodal points in polar coordinates $(\rho, \theta)$ could be expressed as follows [1]:

$$
\{N\}=\frac{1}{\left(R_{2}-R_{1}\right)\left(\theta_{2}-\theta_{1}\right)}\left\{\begin{array}{l}
\left(R_{2}-\rho\right)\left(\theta_{2}-\theta\right) \\
\left(\rho-R_{1}\right)\left(\theta_{2}-\theta\right) \\
\left(\rho-R_{1}\right)\left(\theta-\theta_{1}\right) \\
\left(R_{2}-\rho\right)\left(\theta-\theta_{1}\right)
\end{array}\right\},
$$

where $R_{1}, R_{2}, \theta_{1}, \theta_{2}$ are the values of sector radius and the angle boundary.

And the matrix $\left[B_{i}\right]$ for the $i$-th node of the element is [1]

$$
\left[B_{i}\right]=\left[\begin{array}{cc}
\frac{\partial N_{i}}{\partial \rho} & 0 \\
\frac{N_{i}}{\rho} & \frac{1}{\rho} \frac{\partial N_{i}}{\partial \theta} \\
\frac{1}{\rho} \frac{\partial N_{i}}{\partial \theta} & \frac{\partial N_{i}}{\partial \rho}-\frac{N_{i}}{\rho}
\end{array}\right], \quad i=1,4 .
$$

Analogously the matrix $\left[B_{E i}\right]$ for the $i$-th node is of the same form [16]

$$
\left[B_{E i}\right]=\left[\begin{array}{c}
\frac{\partial N_{i}}{\partial \rho} \\
\frac{1}{\rho} \frac{\partial N_{i}}{\partial \theta}
\end{array}\right], \quad i=1,4 .
$$


Thus, the expression for the stiffness matrix of the sector-type finite element becomes

$$
[K]=h \int_{\theta_{1}}^{\theta_{2}} \int_{R_{1}}^{R_{2}}[B]^{T}\left[c^{E}\right][B] \rho \mathrm{d} \rho \mathrm{d} \theta,
$$

where $h$ is thickness of the element.

Analogously, formulas (7)-(10) of the other matrices have the same structure.

The structural matrices of the sector-type finite element were derived using the computer algebra system VIBRAN, that is a FORTRAN preprocessor for analytical perturbation with polynomials, rational functions, and trigonometric series [3]. A special VIBRAN procedure can generate an optimized FORTRAN code from the obtained analytical expressions [2], which can be directly used in the programs for numerical analysis. The computer algebra technique is more convenient than numerical for this purpose, because the polynomials under integrals are of the negative power of order -1 for the sector-type finite element in the polar coordinates. Consequently, numerical integration is not very useful. Table 1 illustrates the number of products necessary to form the structural matrices of the piezoceramic sector finite element. For the sake of clarity, we consider only three matrices of the mass, stiffness, and electro-elasticity, because their formation is more expensive.

Table 1. The number of products to form the sector-type element matrices

\begin{tabular}{ccc}
\hline Matrix & Numerical integration & Computer Algebra \\
\hline$K$ & 1296 & 820 \\
$T$ & 972 & 634 \\
$M$ & 2792 & 926 \\
Total: & 5060 & 2380 \\
\hline
\end{tabular}

Another geometrically suitable finite element is triangle, which allows modelling various surfaces [17]. The first member of a triangular membrane or a bending element family is the element with three nodes and the interpolating polynomial of second order. The triangular finite element is also suitable to determine the configuration of optimal electrodes of the piezoceramic actuators [4. The interpolating functions for the triangular finite element are expressed in the area of $L$-coordinates [5, 17]

$$
\begin{aligned}
& \left\{N_{i}\right\}=\left\{\begin{array}{c}
L_{1}+L_{1}^{2} L_{2}+L_{1}^{2} L_{3}-L_{1} L_{2}^{2}-L_{1} L_{3}^{2} \\
b_{2}\left(L_{3} L_{1}^{2}+0.5 L_{1} L_{2} L_{3}\right)-b_{3}\left(L_{1}^{2} L_{2}+0.5 L_{1} L_{2} L_{3}\right) \\
c_{2}\left(L_{3} L_{1}^{2}+0.5 L_{1} L_{2} L_{3}\right)-c_{3}\left(L_{1}^{2} L_{2}+0.5 L_{1} L_{2} L_{3}\right)
\end{array}\right\}, \\
& i=1,2,3
\end{aligned}
$$

where $b_{i}, c_{i}$ are nodal coordinate differences with cyclic permutation of indices $1 \rightarrow 2 \rightarrow 3 \rightarrow 1$ for the remaining coefficients [5]. The matrix $\left[B_{i}\right]$ of the triangular element for the $i$-th node is 17 . 


$$
\left[B_{i}\right]=\left[\begin{array}{c}
-\frac{\partial^{2}}{\partial x^{2}} N_{i} \\
-\frac{\partial^{2}}{\partial^{2} y} N_{i} \\
\frac{\partial^{2}}{\partial x \partial y} N_{i}
\end{array}\right], \quad i=1,2,3 .
$$

Thus, the expression of the stiffness matrix for the triangular finite element becomes like this

$$
[K]=h \int_{0}^{1} \int_{0}^{1-L_{1}}[B]^{T}\left[c^{E}\right][B] \mathrm{d} L_{2} \mathrm{~d} L_{1},
$$

where $h$ is thickness of the element.

Analogously, the formulas (7)-(10) of the other matrices are of the same structure. Integration of the products (17) in algebraic form over the area of a finite element in $L$-coordinates according to [17] could be expressed as follows:

$$
\int_{\Delta} L_{1}^{n} L_{2}^{m} L_{3}^{k} \mathrm{~d} \Delta=\frac{n ! m ! k !}{(n+m+k+2) !} 2 \Delta,
$$

where $2 \Delta$ is the triangle area.

Table 2 illustrates the number of products necessary to form the structural matrices of the piezoceramic triangular finite element.

Table 2. The number of products used to form triangular element matrices

\begin{tabular}{ccc}
\hline Matrix & Numerical integration & Computer Algebra \\
\hline$K$ & 2430 & 1204 \\
$T$ & 1660 & 946 \\
$M$ & 3240 & 794 \\
Total: & 7330 & 2944 \\
\hline
\end{tabular}

\section{Conclusions}

The proposed analytical formation of the structural matrices of piezoceramic finite elements reduces the number of floating point operations more than twice, particularly for the mass matrix of the elements. The highest economy is gained during the integration procedure, because, after the integration, the expressions of matrix elements become shorter due to disappearance of variables. The distributed mass, electro-elasticity, and damping matrices always yield relatively simple final analytical expressions even for complex finite elements. Due to the fact that the integral expressions to be integrated, in these cases, have the power at least twice higher than that of the ordinary stiffness matrix, the use of computer algebra remarkably saves the required computer time as compared to the numerical integration technique. It should be also emphasized that the computer time economy is followed by an increased accuracy of computations, which is, in many cases, the most important gain achieved. 


\section{References}

1. Bansevicius, R., Barauskas, R., Kulvietis, G., Ragulskis, K.: Vibromotors for Precision Microrobots. Hemisphere Publishing Corp., USA (1988)

2. Bansevicius, R., Cepulkauskas, A., Kulvietiene, R., Kulvietis, G.: Computer Algebra for Real-Time Dynamics of Robots with Large Number of Joints, Lecture Notes in Computer Science, Vol. 3039. Springer-Verlag, Berlin Heidelberg New York (2004) 278-285

3. Bansevicius, R., Parkin, R., Jebb, A., Knight, J.: Piezomechanics as a Sub-System of Mechatronics: Present State of the Art, Problems, Future Developments. IEEE Transactions on Industrial Electronics, vol. 43 (1) (1996) 23-30.

4. Bansevicius, R., Kulvietis, G., Mazeika, D.: Optimal Electrodes Configuration of Piezodrives. Solid Mechanics and Its Applications, N 73, Kluwer Academic Publ. (2000) 77-83

5. Belevicius, R., Pedersen, P.: Analysis and Sensitivity Analysis by Computer Algebra for a Third Order Plate Finite Element. Comp. \& Struct. 49 (1993) 243-252

6. Besseling, J.F.: Finite Element Properties, Based upon Elastic Potential Interpolation. Hybrid and Mixed Finite Element Meth. Int. Symp. Atlanta, 1981. John Wiley, Chichester (1983) 253-266

7. Han, J.M., Adriaens, T.A., Willem, L., de Koning, Reinder Banning: Modelling Piezoelectric Actuators. IEEE/ASME Transactions on Mechatronics, Vol. 5 (4) (2000) 331-337

8. Laouafa, F., Royis, P.: An Iterative Algorithm for Finite Element Analysis. Journal of Computational and Applied Mathematics, Vol. 164-165 (2004) 469-491

9. Liew, KM, He, XQ, Kitipornchai, S.: Finite Element Method for the Feedback Control of FGM Shells in the Frequency Domain via Piezoelectric Sensors and Actuators. Computer Methods in Applied Mechanics and Engineering, Vol. 193 Issue 3 (2004) 257-273

10. Marczak, R.J.: Object-Oriented Numerical Integration - a Template Scheme for FEM and BEM Applications. Advances in Engineering Software, In Press, Corrected Proof, Available online 11 July 2005

11. Oliveira, A., Sousa P., Costa Branco, P.J.: Surface Deformation by Piezoelectric Actuator: from Park and Agrawal Models to a Simplified Model Formulation Sensors and Actuators. A: Physical, Vol. 115 (2-3) (2004) 235-244

12. Parashar, S., Das Gupta, A., von Wagner, U. \& Hagedorn, P.: Non-linear Shear Vibrations of Piezoceramic Actuators International Journal of Non-Linear Mechanics, 40 (2005) 429-443

13. Samal, M.K. and oths.: A Finite Element Model for Nonlinear Behaviour of Piezoceramics under Weak Electric Fields Finite Elements in Analysis and Design, Vol. 41 (15) (2005) 1464-1480

14. Storck, Heiner, Wallaschek, Jörg: The Effect of Tangential Elasticity of the Contact Layer between Stator and Rotor in Travelling Wave Ultrasonic Motors. International Journal of Non-Linear Mechanics 38 (2003) 143-159

15. Tenchev, R.T.: A Study of the Accuracy of some FEM Stress Recovery Schemes for 2D Stress Concentration Problems. Finite Elements in Analysis and Design, Vol. 29 (2) (1998) 105-119

16. Tzou, H.S.: Piezoelectric Shells (Distributed Sensing and Control of Continua), Kluwer Academic Publishers (1993)

17. Zienkiewicz, O.C., Taylor, R.L.: The Finite Element Method. McGraw-Hill (1991) 